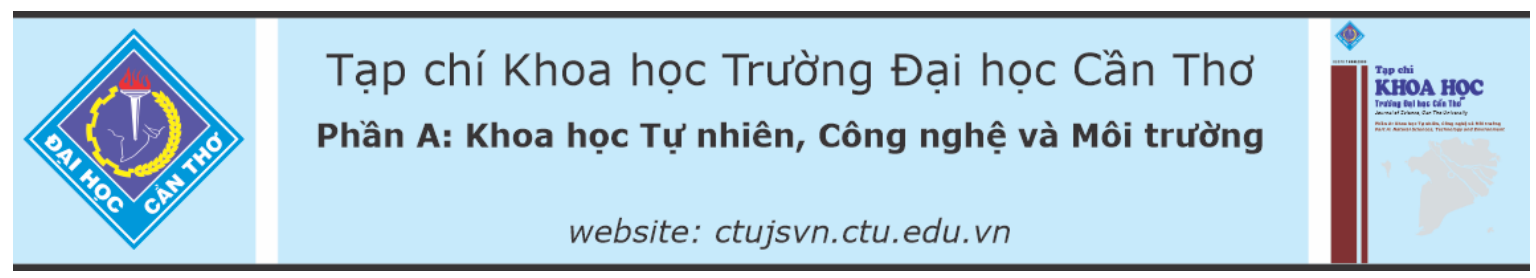

DOI:10.22144/ctu.jvn.2021.173

\title{
TÍNH CHẤT ĐIỆN TỬ CỦA HEXAGONAL CHROMIUM NITRIDE
}

\author{
Nguyễn Thị Kim Quyên ${ }^{1,2 *}$, Phạm Nguyễn Hữu Hạnh ${ }^{3}$ và Vũ Thanh Trà \\ ${ }^{1}$ Khoa Kỹ thuật Công nghệ, Truoòng Đại học Kiên Giang \\ ${ }^{2}$ Khoa Sau đại họ, Truoòng Đại họ Cần Tho \\ ${ }^{3}$ Truoòng THCS \& THPT Thới Thuận, Cần Tho \\ ${ }^{4}$ Bộ môn Su phạm Vật lý, Khoa Su phạm, Truòng Đại hoc Cần Tho \\ *Nguoòi chịu trách nhiệm về bài viết: Nguyễn Thị Kim Quyên (email: kimquyen929@gmail.com)
}

\section{Thông tin chung:}

Ngày nhận bài: $10 / 11 / 2021$

Ngày nhận bài sủa: 11/12/2021

Ngày duyệt đăng: 25/12/2021

Title:

The electronic structure of hexagonal chromium nitride

\section{Tù khóa:}

Cấu trúc vùng năng lượng, cấu trúc vênh, Chromium Nitride, phương pháp gần đúng liên kết manh

\section{Keywords:}

Band structure, buckled structure, Chromium Nitride, tight binding model

\begin{abstract}
In this study, the Hamiltonian model was built by using the tight binding (TB) calculation that computes the electronic properties of hexagonal chromium nitride ( $h-C r N)$ for planar and buckled structures. Based on the results obtained and by comparison with the other results from the similar model such as the ab initio method on different structures and identified the structural parameters for the nearest neighborhood interactions of the atoms. In addition, this paper also shows that the h-CrN exhibits metallicity of thin honeycomb materials in flat and buckled states. However, the electronic structure of the material in the buckled state has changed more pronounced than the one in planar structure, which predicts interesting variations of the electronic distributions and its properties under the influence of external stimuli as well as the applicability to future spintronic technology.
\end{abstract}

\section{TÓM TẮT}

Trong nghiên cứu này, phuơng pháp gần đúng liên kết mạn (TB) được sử dụng để xây dưng mô hình Hamiltonian tính toán đặc trung điện tư của hexagonal chromium nitride $(h-C r N)$ cho cấu trúc phẳng và nhấp nhô. Tùr kết quả tính toán thu được, đồng thời so sánh với kết quả tính toán tù mô hìn tuoong tụ theo phuoong pháp ab initio trên các cấu trúc khác nhau, bộ tham số cấu trúc cho các tuoong tác lân cận bậc một của các nguyên tử cấu thành vật liệu được xác định. Ngoài ra, kết quả cũng chỉ ra rằng ở trạng thái phăng và nhấp nhô, h-CrN thể hiện tính chất kim loại của vật liệu mỏng dạng tổ ong. Tuy nhiên, cấu trúc điện tư vật liệu ở trạng thái nhấp nhô có nhiều thay đổi hơn so với cấu trúc phẳng, dụ đoán nhũng thay đổi thú vị về tính chất điện của vật liệu duoới tác động của kích thích bên ngoài cũng nhu khả năng úng dụng vào công nghệ spintronic trong tuong lai.

\section{GIỚI THIỆ}

Ngày nay, vật liệu mỏng hai chiều như graphene và tựa graphene đang nhận được sự quan tâm đặc biệt của các nhà nghiên cứu lý thuyết và thực nghiệm (Guzmán-Verri et al., 2007; Neto et al., 2009; Novoselov et al., 2012; Novoselov et al., 2004; Takeda et al., 1994; ), bởi các đặc tính vật lý 
thú vị của nhóm vật liệu này và tiềm năng ứng dụng mạnh mẽ trong công nghiệp bán dẫn và transistor. Tuy nhiên, đối với các ứng dụng công nghiệp liên quan đến sự phân cực của spin thì nhóm các vật liệu hai chiều có cấu trúc là một lớp nguyên tử này lại không phải là đối tượng hàng đầu nhận được sự quan tâm. Bởi đây là nhóm vật liệu không có hoặc có từ tính rất yếu (Ali et al.,2017; Zheng et al., 2012). Trong khi đó, các vật liệu ở nhóm kim loại chuyển tiếp (transition metal nitrides-TMNs) hoàn toàn có thể khắc phục được nhược điểm của nhóm vật liệu hai chiều này. Ngoài những tính chất vật lý giống với nhóm vật liệu hai chiều, các vật liệu thuộc TMNs còn có khả năng hình thành các trạng thái spin bề mặt (do cấu trúc vật liệu tồn tại electron ở phân lớp $d$ ), rất thuận lợi cho việc ứng dụng vào các thiết bị spintronic.

Trong số đó, với cấu trúc tinh thể được coi là một thành viên của hệ TMNs trong khi cấu trúc theo hướng (111) lại là một màng mỏng như một lớp màng tổ ong của nhóm vật liệu 2D (dạng hexagonal), Chromium Nitride $(\mathrm{CrN})$ có thể nói là sự kết hợp hoàn hảo của những tính chất đặc biệt của 2 hệ vật liệu lớn này (Kuklin et al.,2016; Luo et al., 2017), hứa hẹn nhiều ứng dụng cho ngành công nghiệp điện tử. Tuy nhiên, số lượng nghiên cứu lý thuyết về vật liệu này còn khá khiêm tốn, đa phần tập trung ở các phương pháp thực nghiệm. Do đó, nhu cầu về việc phát triển nghiên cứu lý thuyết của vật liệu này là điều thực sự cần thiết để có thể dự đoán những tính chất đặc biệt của vật liệu dưới các tác nhân kích thích bên ngoài cũng như lựa chọn những khả năng để đưa chúng vào ứng dụng.

Trong nghiên cứu này, bằng việc sử dụng phương pháp gần đúng liên kết mạnh để xây dựng mô hình tính toán cho các tương tác lân cận gần nhất của các orbital nguyên tử, bài toán cấu trúc vùng của vật liệu $\mathrm{CrN}$ được khảo sát cho cả hai trạng thái: trạng thái phẳng và trạng thái nhấp nhô; đồng thời, so sánh với kết quả thu được từ các nghiên cứu trước để đề xuất bộ tham số cấu trúc phù hợp, hướng đến việc sử dụng trong các tính toán tiếp theo, liên quan đến các tương tác spin của vật liệu. Vì thế, đây có thể là nghiên cứu mở đường cho chuỗi những nghiên cứu sau này về nhóm các vật liệu đang có giá trị ứng dụng vào cuộc cách mạng công nghiệp như trong thời điểm hiện nay.

\section{MÔ HÌNH VÀ PHƯƠNG PHÁP TÍNH TOÁN}

\subsection{Mô hình}

Để khảo sát bài toán cấu trúc vùng của vật liệu, tấm h-CrN được chia thành các ô đơn vị, sử dụng phương pháp gần đúng liên kết mạnh (Zhang et al., 2018) với cách tính năng lượng tương tác như thể hiện ở Hình 1 . Khi đó, các nguyên tử $\mathrm{Cr}$ và $\mathrm{N}$ được biểu diễn lần lượt bằng các hạt màu đỏ thẫm và đen (thể hiện trong Hình 1a). Mô hình tính toán tương tác giữa các orbital trên phân lớp $d$ và $p$ được tập trung xây dựng, đại diện tương ứng cho nguyên tử Cr và $\mathrm{N}$. Tuy nhiên, một điều thú vị là mặc dù các orbital cùng loại sẽ mô tả cùng trạng thái lượng tử của nguyên tử, nhưng do sự sắp xếp xen kẽ của nguyên tử trong cấu trúc $\mathrm{h}$-CrN đã dẫn đến sự xen phủ giữa các orbital này theo phương diện hình học là hoàn toàn khác nhau. Do đó, để thuận tiện trong tính toán, các tương tác được chia thành trực diện và xiên nhau giữa các orbital nguyên tử cùng loại. Cụ thể, Hình $1 \mathrm{~b}$ cho thấy đối với orbital $d_{x^{2} y^{2}}$, sự xen phủ giữa các orbital chia thành 2 loại tương tác là

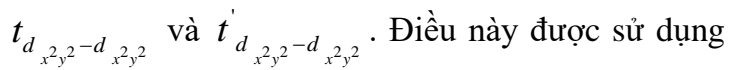
để tính toán tương tự cho các orbital khác và được thống kê cụ thể như Bảng 1 .

\section{Bảng 1. Quy ước các tham số tương tác giữa các orbital nguyên tử}

\begin{tabular}{ccc}
\hline Tương tác giữa các orbital & Năng lượng tương tác trực diện & Năng lượng tương tác xiên \\
\hline$d_{x z}-d_{x z}$ & $t_{d d \delta}$ & $t_{d d \delta}^{\prime}$ \\
$d_{y z}-d_{y z}$ & $t_{d d \pi}$ & $t_{d d \pi}^{\prime}$ \\
$d_{x^{2} y^{2}}-d_{x^{2} y^{2}}$ & $t_{d d \sigma}$ & $t_{d d \sigma}^{\prime}$ \\
$d_{x y}-d_{x y}$ & $t_{d d \pi^{*}}$ & $t_{d d \pi^{*}}^{\prime}$ \\
$d_{z^{2}}-d_{z^{2}}$ & $t_{d d \pi \|}$ & $t_{d d \pi \|}^{\prime}$ \\
$p_{x}-p_{x}$ & $t_{p p \pi}$ & $t_{p p \pi}^{\prime}$ \\
$p_{y}-p_{y}$ & $t_{p p \sigma}$ & $t_{p p \sigma}^{\prime}$ \\
$p_{z}-p_{z}$ & $t_{p p \pi \|}$ & $t_{p p \pi \|}^{\prime}$
\end{tabular}


(a)
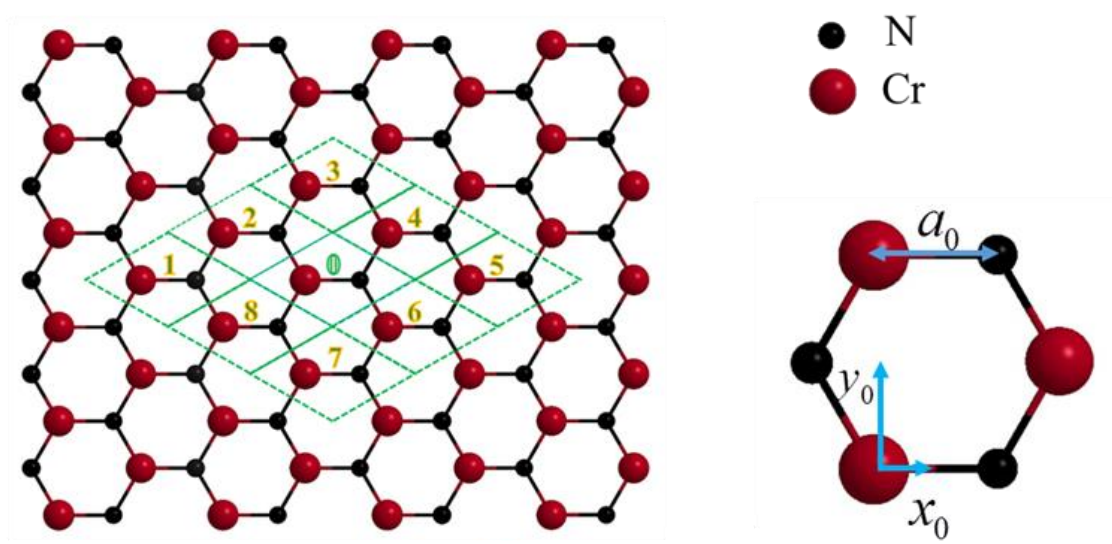

(b)
$t_{d_{x^{2} y^{2}}-d_{x^{2} y^{2}}}$

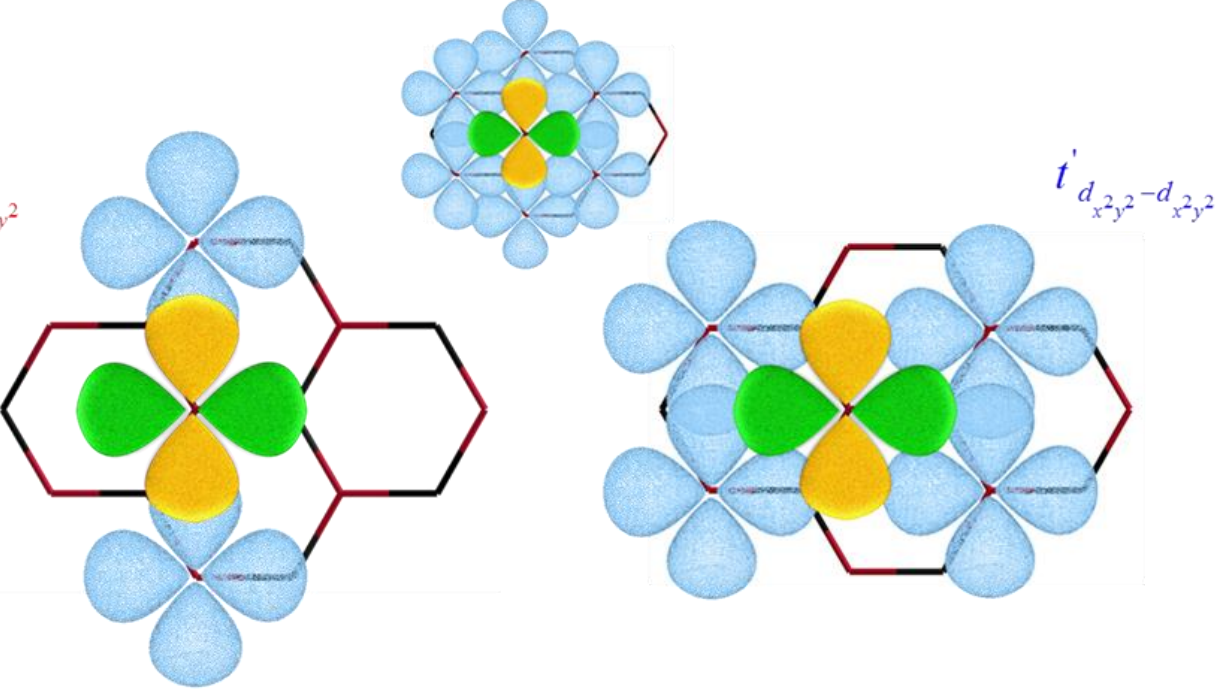

Hình 1. (a) Mô hình tính toán h-CrN dựa trên phương pháp gần đúng liên kết mạnh, (b) Tương tác giữa các orbital $d_{x^{2} y^{2}}$ của nguyên tử $\mathrm{Cr}$

\subsection{Phương pháp tính toán}

Để tính toán các đặc trưng dẫn của vật liệu cũng như đưa ra ảnh hưởng của các tham số cấu trúc lên cấu trúc vùng năng lượng của vật liệu, phương pháp gần đúng liên kết mạnh được sử dụng, tính toán cho các nguyên tử lân cận bậc 1 , thể hiện trong phương trình (1) (Zhang et al., 2018):

$$
H(k)=H_{h o p}(k)+H_{S O C}-H_{M}=\left[\begin{array}{cc}
H_{h o p} & 0 \\
0 & H_{h o p \downarrow}
\end{array}\right]+\left[\begin{array}{cc}
H_{S O C \uparrow} & 0 \\
0 & H_{S O C \downarrow}
\end{array}\right]-\left[\begin{array}{cc}
H_{M \uparrow} & 0 \\
0 & H_{M \downarrow}
\end{array}\right]
$$

Phương trình (1) thể hiện Hamiltonian tổng, gồm 3 thành phần cụ thể như sau:

Thành phần thứ 1: Thể hiện năng lượng tương tác giữa các orbital nguyên tử cùng loại gần nhau nhất. Tuỳ theo trạng thái của vật liệu (phẳng hoặc nhấp nhô) mà mô hình tính toán $H_{h o p}$ sẽ thay đổi khác nhau, được trình bày cụ thể ở phần sau.

Thành phần thứ 2: Đặc trưng cho tương tác spin (spin orbit coupling - SOC) của các orbital nguyên tử, được biểu diễn bởi ma trận (2) (Konschuh et al., 2010): 


$$
H_{S O C \uparrow \downarrow}=S\left[\begin{array}{cccccccc}
0 & -i \lambda_{C r} & -i \lambda_{C r} & i \lambda_{C r} & i \sqrt{3} \lambda_{C r} & 0 & 0 & 0 \\
i \lambda_{C r} & 0 & -i \lambda_{C r} & -i \lambda_{C r} & -i \sqrt{3} \lambda_{C r} & 0 & 0 & 0 \\
i \lambda_{C r} & i \lambda_{C r} & 0 & -2 i \lambda_{C r} & 0 & 0 & 0 & 0 \\
-i \lambda_{C r} & i \lambda_{C r} & 2 i \lambda_{C r} & 0 & 0 & 0 & 0 & 0 \\
-i \sqrt{3} \lambda_{C r} & i \sqrt{3} \lambda_{C r} & 0 & 0 & 0 & 0 & 0 & 0 \\
0 & 0 & 0 & 0 & 0 & 0 & -i \lambda_{N} & i \lambda_{N} \\
0 & 0 & 0 & 0 & 0 & i \lambda_{N} & 0 & -i \lambda_{N} \\
0 & 0 & 0 & 0 & 0 & -i \lambda_{N} & i \lambda_{N} & 0
\end{array}\right]
$$

Với $s= \pm 1$ tương ứng với thành phần spin up/down; $\lambda_{C r}, \lambda_{N}$ lần lượt là độ lớn của SOC tương ứng cho nguyên tử $\mathrm{Cr}$ và $\mathrm{N}$.

Thành phần thứ 3 : Ảnh hưởng từ trường, được biểu diễn như ở phương trình (3). Trong đó $M_{i}$ là

$$
H_{M \uparrow / \downarrow}= \pm\left[\begin{array}{cccccccc}
M_{C r} & 0 & 0 & 0 & 0 & 0 & 0 & 0 \\
0 & M_{C r} & 0 & 0 & 0 & 0 & 0 & 0 \\
0 & 0 & M_{C r} & 0 & 0 & 0 & 0 & 0 \\
0 & 0 & 0 & M_{C r} & 0 & 0 & 0 & 0 \\
0 & 0 & 0 & 0 & M_{C r} & 0 & 0 & 0 \\
0 & 0 & 0 & 0 & 0 & M_{N} & 0 & 0 \\
0 & 0 & 0 & 0 & 0 & 0 & M_{N} & 0 \\
0 & 0 & 0 & 0 & 0 & 0 & 0 & M_{N}
\end{array}\right]
$$

một nguyên tử $\mathrm{Cr}$ và một nguyên tử $\mathrm{N}$ (thể hiện trên Hình $1 \mathrm{a}$ ), tính toán cho lân cận gần nhất của các nguyên tử. Khi đó, dựa trên trạng thái vật liệu khác nhau (phẳng hoặc nhấp nhô), ma trận $H_{\text {hop }}$ được cụ thể hoá như sau:

Ở trạng thái phẳng: Tính toán thực hiện trên tương tác giữa các orbital cùng loại và tính thêm tương tác giữa orbital $d_{x^{2} y^{2}}$ của Cr và orbital $p_{x}$ của N. Khi đó, $H_{\text {hop }}$ được biểu diễn thông qua ma trận $8 \times 8$, với vị trí của các orbital được sắp xếp lần lượt là $d_{x z}, d_{y z}, d_{x^{2} y^{2}}, d_{x y}, d_{z^{2}}, p_{x}, p_{y}, p_{z}$, như trình bày ở (5). 


$$
H_{\text {hop } \uparrow \downarrow}=\left[\begin{array}{cccccccc}
H_{11} & 0 & 0 & 0 & 0 & 0 & 0 & 0 \\
0 & H_{22} & 0 & 0 & 0 & 0 & 0 & 0 \\
0 & 0 & H_{33} & 0 & 0 & H_{36} & 0 & 0 \\
0 & 0 & 0 & H_{44} & 0 & 0 & 0 & 0 \\
0 & 0 & 0 & 0 & H_{55} & 0 & 0 & 0 \\
0 & 0 & H_{63} & 0 & 0 & H_{66} & 0 & 0 \\
0 & 0 & 0 & 0 & 0 & 0 & H_{77} & 0 \\
0 & 0 & 0 & 0 & 0 & 0 & 0 & H_{88}
\end{array}\right]
$$

Trong đó:

$$
\begin{aligned}
& H_{11}=\varepsilon_{d_{x z}}+t_{d d \delta} \cos \left(2 y_{0} k_{y}\right)+t_{d d \delta}^{\prime} \cos \left(3 x_{0} k_{x}\right) \cos \left(y_{0} k_{y}\right) \\
& H_{22}=\varepsilon_{d_{y z}}+t_{d d \pi} \cos \left(2 y_{0} k_{y}\right)+t_{d d \pi}^{\prime} \cos \left(3 x_{0} k_{x}\right) \cos \left(y_{0} k_{y}\right) \\
& H_{33}=\varepsilon_{x_{x^{2} y^{2}}}+t_{d d \sigma} \cos \left(2 y_{0} k_{y}\right)+t_{d d \sigma}^{\prime} \cos \left(3 x_{0} k_{x}\right) \cos \left(y_{0} k_{y}\right) \\
& H_{44}=\varepsilon_{d_{x y}}+t_{d d \pi^{*}} \cos \left(2 y_{0} k_{y}\right)+t_{d d \pi^{*}}^{\prime} \cos \left(3 x_{0} k_{x}\right) \cos \left(y_{0} k_{y}\right) \\
& H_{55}=\varepsilon_{d_{z^{2}}}+t_{d d \pi \|} \cos \left(2 y_{0} k_{y}\right)+t_{d d \pi \|}^{\prime} \cos \left(3 x_{0} k_{x}\right) \cos \left(y_{0} k_{y}\right) \\
& H_{66}=\varepsilon_{p_{x}}+t_{p p \pi} \cos \left(2 y_{0} k_{y}\right)+t_{p p \pi}^{\prime} \cos \left(3 x_{0} k_{x}\right) \cos \left(y_{0} k_{y}\right) \\
& H_{77}=\varepsilon_{p_{y}}+t_{p p \sigma} \cos \left(2 y_{0} k_{y}\right)+t_{p p \sigma}^{\prime} \cos \left(3 x_{0} k_{x}\right) \cos \left(y_{0} k_{y}\right) \\
& H_{88}=\varepsilon_{p_{z}}+t_{p p \pi \|} \cos \left(2 y_{0} k_{y}\right)+t_{p p \pi \|}^{\prime} \cos \left(3 x_{0} k_{x}\right) \cos \left(y_{0} k_{y}\right) \\
& H_{36}=t_{d_{x^{2} y^{2}}-p_{x}} \cdot\left(\exp \left(i\left(-k_{x} x_{0}-k_{y} y_{0}\right)\right)+\exp \left(i\left(-k_{x} x_{0}+k_{y} y_{0}\right)\right)\right) \\
& H_{63}=H_{36}{ }^{\dagger}
\end{aligned}
$$

Với: $\varepsilon_{i}$ là năng lượng nội tại (onsite energy) của các orbital nguyên tử, $t_{i j}$ là năng lượng tương tác giữa các orbital nguyên tử, quy ước cụ thể như trong Bảng $1 ; x_{0}$ và $y_{0}$ là khoảng cách giữa hai orbital nguyên tử theo phương $x$ và $y$, cụ thể là: $x_{0}=\frac{a_{0}}{2}$ và $y_{0}=\frac{a_{0} \sqrt{3}}{2}$, với $a_{0}:$ khoảng cách giữa hai nguyên tử Cr-N gần nhau nhất.
Ở trạng thái nhấp nhô: Dựa trên bài toán dạng phẳng ban đầu, mô hình bổ sung thêm một số tương tác khác giữa các orbital khác nhau của nguyên tử, nhu:

$d_{x z}-d_{x^{2} y^{2}}, d_{x z}-d_{x y}, d_{y z}-d_{x^{2} y^{2}}, d_{y z}-d_{x y}, d_{x z}-p_{x}$

. Khi đó, Hamiltonian $H_{\text {hop }}$ trong phương trình được viết lại cho mô hình nhấp nhô, có dạng cụ thể như sau: 


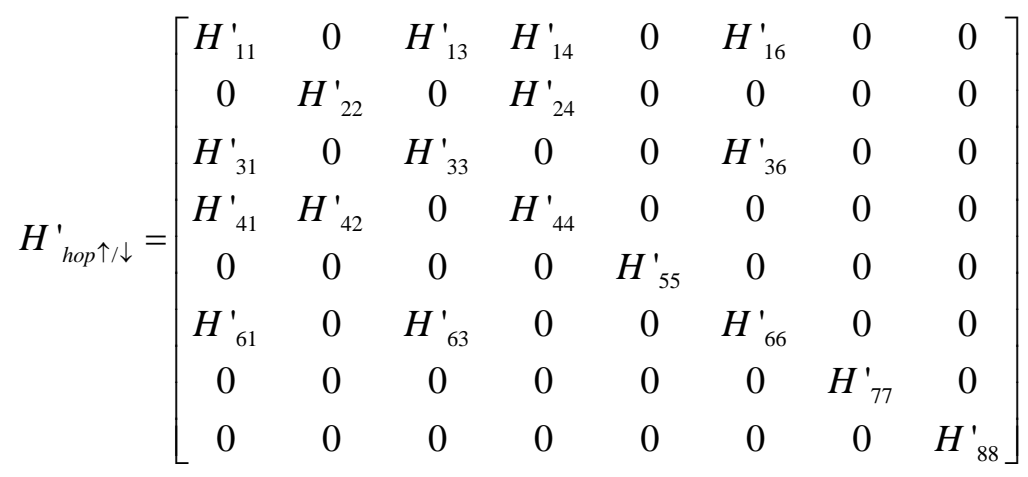

Trong đó:

$$
\begin{aligned}
& H_{13}^{\prime}=t_{d_{x z}-d_{x^{2} y^{2}}} \cos \left(2 y_{0} k_{y}\right)+t_{d_{x z}-d_{x^{2} y^{2}}} \cos \left(3 x_{0} k_{x}\right) \cos \left(y_{0} k_{y}\right) \\
& H_{14}^{\prime}=t_{d_{x z}-d_{x y}} \cos \left(2 y_{0} k_{y}\right)+t_{d_{x z}-d_{x y}} \cos \left(3 x_{0} k_{x}\right) \cos \left(y_{0} k_{y}\right) \\
& H^{\prime}{ }_{24}=t_{d_{y z}-d_{x y}} \cos \left(2 y_{0} k_{y}\right)+t_{d_{y z}-d_{x y}} \cos \left(3 x_{0} k_{x}\right) \cos \left(y_{0} k_{y}\right) \\
& H_{16}^{\prime}=t_{d_{x z}-p_{x}} \cdot\left(\exp \left(i\left(-k_{x} x_{0}-k_{y} y_{0}\right)\right)+\exp \left(i\left(-k_{x} x_{0}+k_{y} y_{0}\right)\right)\right)+t_{d_{x z}-p_{x}}^{\prime} \exp \left(i\left(2 k_{x} x_{0}\right)\right) \\
& H_{31}^{\prime}=\left(H_{13}^{\prime}\right)^{\dagger}, H_{41}^{\prime}=\left(H_{14}^{\prime}\right)^{\dagger}, H_{42}^{\prime}=\left(H_{24}^{\prime}\right)^{\dagger}, H_{61}^{\prime}=\left(H_{16}^{\prime}\right)^{\dagger}
\end{aligned}
$$

Như vậy, với việc xây dựng các Hamilton thành phần khác nhau khi tính tương tác giữa các orbital nguyên tử, ảnh hưởng của các tham số cấu trúc lên tính chất điện tử của vật liệu được khảo sát và thu được kết quả trình bày ở phần 3 .

(a)
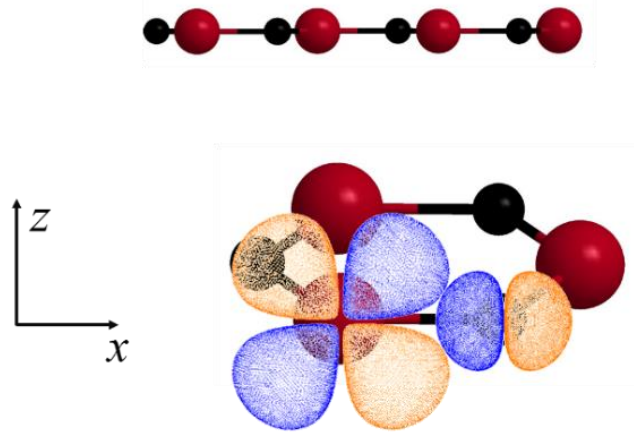

\section{KẾT QUẢ VÀ THẢO LUẬN}

3.1. Bài toán cấu trúc vùng năng lượng của h-CrN ở trạng thái phẳng (b)

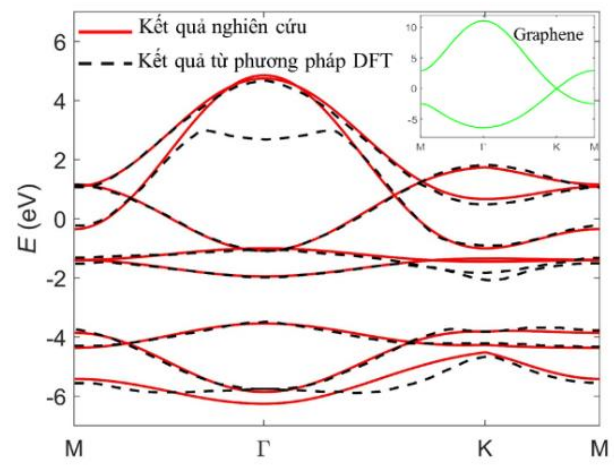

Hình 2. (a) Tấm h-CrN ở dạng phẳng và mối liên hệ giữa orbital $d_{x z}-p_{x}$ của $\mathrm{Cr}$ và $\mathrm{N}$; (b): Cấu trúc vùng năng lượng của cấu trúc $\mathrm{h}-\mathrm{CrN}$ dạng phẳng (đường màu đỏ) so sánh với kết quả nghiên cứu trước từ phương pháp DFT (đường màu đen) (Luo et al., 2017)

Trong bài toán này, đầu tiên phương pháp gần đúng liên kết mạnh được sử dụng tính toán cho mô hình phẳng của h-CrN với $\lambda_{C r}=\lambda_{N}=0$,
$M_{C r}=M_{N}=0$ và chỉ khảo sát theo tương tác của các orbital nguyên tử $\mathrm{Cr}-\mathrm{Cr}$ và $\mathrm{N}-\mathrm{N}$ gần nhau nhất với các tham số tương tác tương ứng như thể hiện trong Bảng 2. 
Bảng 2. Các tham số tương tác của các orbital nguyên tử trong cấu trúc h-CrN dạng phẳng

\begin{tabular}{lccccc}
\hline & $d_{x z}$ & $d_{y z}$ & $d_{x^{2} y^{2}}$ & $d_{x y}$ & $d_{z^{2}}$ \\
\hline$\varepsilon_{i}(e V)$ & 0,80 & $-1,30$ & 2,00 & 0,95 & $-1,55$ \\
$t(e V)$ & $-0,76$ & 0,10 & 0,86 & 1,30 & $-0,12$ \\
$t^{\prime}(e V)$ & $-1,12$ & 0,20 & 1,84 & 2,60 & $-0,28$ \\
& $p_{x}$ & $p_{y}$ & $p_{z}$ & $d_{x^{2} y^{2}}-p_{x}$ & \\
$\varepsilon_{i}(e V)$ & $-4,00$ & $-4,50$ & $-5,10$ & & \\
$t(e V)$ & 0,10 & $-0,36$ & $-0,74$ & $-0,30$ & \\
$t^{\prime}(e V)$ & 0,40 & $-1,00$ & $-0,42$ & & \\
\hline
\end{tabular}

Kết quả thể hiện trong Hình $2 b$ cho thấy cấu trúc vùng của vật liệu thu được (đường liền nét màu đỏ) gần như tương ứng với kết quả nghiên cứu trước (đường đứt nét màu đen) (Luo et al., 2017) tại các điểm Dirac $\mathrm{M}, \Gamma, \mathrm{K}$ đặc biệt trong khoảng $\mathrm{M}-\Gamma$ và $\Gamma-\mathrm{K}$. Điều này cho thấy vật liệu $\mathrm{h}-\mathrm{CrN}$ với trạng thái phẳng thể hiện tính chất kim loại khi cấu trúc vùng gần mức Fermi $E_{F}=0$ dường như không tồn tại một độ rộng vùng cấm nội tại. Bên cạnh đó, khảo sát với các tham số tương tác khác nhau, kết quả chỉ ra rằng tương tác của các orbital $d_{x^{2} y^{2}}, d_{x z}$ và $d_{x y}$ có ảnh hưởng quan trọng đến sự sắp xếp điện tử ở các mức năng lượng gần mức Fermi, hay nói cách khác, các tương tác này ảnh hưởng mạnh mẽ đến trạng thái dẫn của vật liệu.

(a)
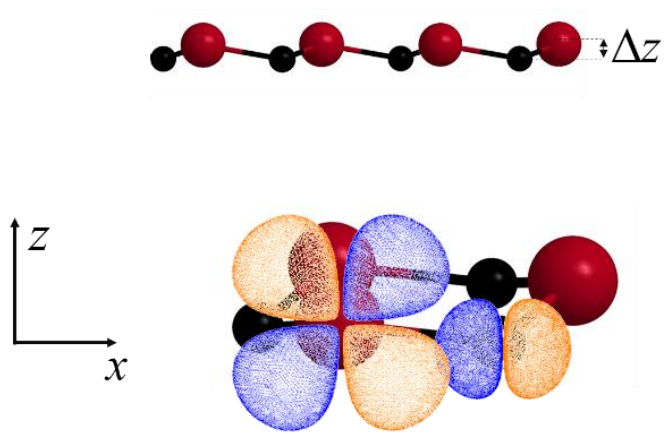

Tuy nhiên, từ Hình $2 b$, kết quả cho thấy rằng hai điểm uốn trong cấu trúc vùng của vật liệu không nằm tại các điểm Dirac, điều này hoàn toàn khác so với kết quả thu được từ một vật liệu dạng tổ ong khác là graphene (thể hiện ở hình chèn bên góc trên bên phải của hình $2 b$ ). Do đó, từ kết quả khảo sát, chúng tôi dự đoán rằng việc tồn tại các electron ở phân lớp $d$ sẽ dẫn đến nhiều tính chất thú vị, đặc biệt sẽ thể hiện mạnh mẽ trong việc tính toán trạng thái spin của vật liệu." Vì thế, với mong muốn đưa ra cái nhìn tổng quát về vật liệu h-CrN, mô hình mở rộng được phát triển tính toán là h-CrN ở trạng thái nhấp nhô của vật liệu, như thể hiện ở Hình 3.

\subsection{Trạng thái nhấp nhô của h-CrN}

(b)

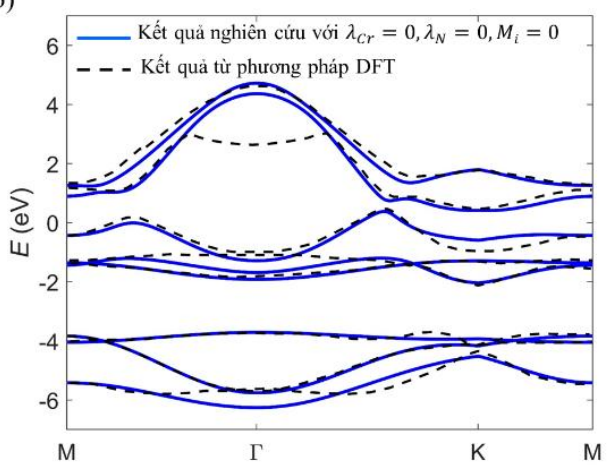

Hình 3. (a) Tấm h-CrN ở dạng nhấp nhô và mối liên hệ giữa orbital $d_{x z}-p_{x}$ của $\mathrm{Cr}$ và $\mathrm{N}$; (b): Cấu trúc vùng năng lượng của cấu trúc h-CrN dạng nhấp nhô (đường màu xanh) so sánh với kết quả nghiên cứu trước từ phương pháp DFT (đường màu đen) (Luo et al., 2017)

Tấm h-CrN khi ở trạng thái nhấp nhô (thể hiện ở Hình $3 a$ ) được xem như hai lớp các nguyên tử $\mathrm{Cr}$ và $\mathrm{N}$ nằm cách nhau một khoảng $\Delta z=0.2 \stackrel{0}{\mathrm{~A}}$ (Luo et al.,
2017) với các nguyên tử Cr nằm ở lớp trên trong khi các nguyên tử $\mathrm{N}$ nằm ở lớp dưới. Như vậy, với trạng thái nhấp nhô của vật liệu khi các nguyên tử $\mathrm{Cr}$ lệch khỏi mặt phẳng ban đầu, sự chồng phủ giữa các 
orbital nguyên tử có sự thay đổi, dẫn đến sự thay đổi về trạng thái liên kết cũng như độ lớn về mặt tương tác giữa chúng. Cụ thể, như thể hiện trên Hình $3 \mathrm{a}$, với sự nhấp nhô của mạng, tương tác giữa orbital $d_{x z}$ của nguyên tử $\mathrm{Cr}$ và orbital $p_{x}$ của nguyên tử $\mathrm{N}$ được hình thành, điều này hoàn toàn khác so với mô hình phẳng, thể hiện trên Hình $2 \mathrm{a}$. Do đó, mô hình $H^{\prime}{ }_{h o p \uparrow / \downarrow}$ xuất hiện một tương tác mới giữa hai nguyên tử là:

$$
H^{\prime}{ }_{16}=t_{d_{x z}-p_{x}} \cdot\left(\exp \left(i\left(-k_{x} x_{0}-k_{y} y_{0}\right)\right)+\exp \left(i\left(-k_{x} x_{0}+k_{y} y_{0}\right)\right)\right)+t_{d_{x z}-p_{x}}^{\prime} \exp \left(i\left(2 k_{x} x_{0}\right)\right)
$$

$H^{\prime}{ }_{h o p \uparrow / \downarrow}$ được sử dụng trong phương trình (6) để tính Hamiltonian tổng, kết hợp với bộ tham số thu được như trình bày trong Bảng $3 \mathrm{a}, \mathrm{b}$, đồng thời với $\lambda_{C r}=\lambda_{N}=0, M_{i}=0$, kết quả về cấu trúc vùng năng lượng của $\mathrm{h}-\mathrm{CrN}$ ở trạng thái nhấp nhô được thể hiện cụ thể trong Hình $3 \mathrm{~b}$.

Bảng 3a. Các tham số tương tác của các orbital cùng loại của các nguyên tử trong cấu trúc h-CrN ở trạng thái nhấp nhô

\begin{tabular}{lcccc}
\hline & $d_{x z}$ & $d_{y z}$ & $d_{x^{2} y^{2}}$ & $d_{x y}$ \\
\hline$\varepsilon_{i}(\mathrm{eV})$ & 0,50 & $-1,20$ & 1,70 & 0,60 \\
$t(\mathrm{eV})$ & $-0,52$ & 0,05 & 0,74 & 1,12 \\
$t^{\prime}(\mathrm{eV})$ & $-0,80$ & 0,10 & 1,48 & 2,24 \\
& $d_{z^{2}}$ & $p_{x}$ & $p_{y}$ & $p_{z}$ \\
$\varepsilon_{i}(\mathrm{eV})$ & $-1,50$ & $-3,70$ & $-4,70$ & $-5,10$ \\
$t(\mathrm{eV})$ & $-0,14$ & 0,03 & $-0,10$ & $-0,74$ \\
$t^{\prime}(\mathrm{eV})$ & $-0,28$ & 0,06 & $-0,96$ & $-0,42$ \\
\hline
\end{tabular}

Bảng 3b. Các tham số tương tác của các loại orbital khác nhau của các nguyên tử trong cấu trúc h-CrN ở trạng thái nhấp nhô

\begin{tabular}{lrrrrrr}
\hline & $d_{x z}-d_{x^{2} y^{2}}$ & $d_{x z}-d_{x y}$ & $d_{y z}-d_{x^{2} y^{2}}$ & $d_{y z}-d_{x y}$ & $d_{x z}-p_{x}$ & $d_{x^{2} y^{2}}-p_{x}$ \\
\hline$t(e V)$ & $-0,56$ & $-0,80$ & $-0,30$ & 0,60 & $-0,65$ & $-0,30$ \\
$t^{\prime}(e V)$ & $-0,88$ & $-0,40$ & $-0,32$ & 1,00 & 0,20 & \\
\hline
\end{tabular}

Kết quả cho thấy rằng với sự hình thành các tương tác mới giữa các orbital nguyên tử, các điểm uốn nằm trong khoảng $M-\Gamma$ và $\Gamma-K$ đã được mở ra, tuy nhiên trong khoảng mức năng lượng gần mức Fermi, độ rộng vùng cấm của vật liệu vẫn bằng 0 , hay nói cách khác, vật liệu vẫn thể hiện tính kim loại của tấm h-CrN ban đầu.

Như vậy, để phần mô tả của bản thảo được rõ ràng hơn. Như vậy, khảo sát cấu trúc vùng năng lượng của vật liệu ở hai trạng thái, kết quả cho thấy rằng ở trạng thái phẳng, điện tử tập trung chủ yếu ở vùng giao nhau giữa các orbital $d_{z^{2}}, d_{y z}$ và $p_{z}$ nơi hình thành các liên kết $\pi$ giữa các orbital nguyên tử. Trong khi đó, ở trạng thái nhấp nhô, mật độ điện tử có xu hướng giảm do sự phá vỡ liên kết $\pi$ đối xứng giữa $d_{z^{2}}$ với $p_{z}, d_{y z}$ với $p_{z}$. Nguyên nhân dẫn đến điều này là khi ở trạng thái nhấp nhô, các nguyên tử $\mathrm{N}$ sẽ nằm ở một mặt phẳng riêng biệt so với các nguyên tử $\mathrm{Cr}$, vì vậy các orbital giữa các nguyên tử $\mathrm{N}$ lân cận nhau sẽ có độ chồng phủ cao hơn, hình thành các liên kết bền hơn so với việc chồng phủ với các orbital của $\mathrm{Cr}$. Bên cạnh đó, các orbital của $\mathrm{Cr}$ cũng hình thành các tương tác mới, dẫn đến việc suy giảm tương tác so với các tương tác ban đầu (ở trạng thái phẳng). Chính kết quả này đã cho thấy trạng thái nhấp nhô của tấm h-CrN có tác dụng góp phần trong việc điều khiển trạng thái dẫn điện của vật liệu ở trạng thái kích thích, hứa hẹn có nhiều tiềm năng trong việc ứng dụng vào thực tế. Ngoài ra, để đưa ra cái nhìn tổng quát về cấu trúc điện tử của vật liệu ở trạng thái nhấp nhô, ảnh hưởng của spin cũng được tiến hành khảo sát và thu được kết quả như Hình 4.

\section{3. Ảnh hưởng của $\mathrm{SOC}$ trong bài toán cấu trúc điện tử của vật liệu}

Hình 4 khảo sát ảnh hưởng của các giá trị SOC khác nhau trong bài toán cấu trúc vùng của vật liệu, 
với $M_{C r}=0,1, M_{N}=0,07$ và các tham số tương tác như đã trình bày trong Bảng $3 \mathrm{a}, \mathrm{b}$. Kết quả khảo sát cho thấy với giá trị $\lambda_{C r}=0,15 \mathrm{eV}$, vị trí của một số mức năng lượng được thay đổi, tuy nhiên dạng chung trong cấu trúc vùng vẫn không thay đổi. Cụ thể là các mức năng lượng thể hiện tương tác của các orbital trên phân lớp $d$ của nguyên tử $\mathrm{Cr}$ nằm gần mức Fermi được tách rời nhau ra, thể hiện ở Hình $4 \mathrm{~b}$. Điều này khác với kết quả thu được ở Hình $4 \mathrm{a}$, khi không có sự hiện diện của $\lambda_{C r}$. Đồng thời, ở Hình $4 \mathrm{c}$, khi đưa giá trị $\lambda_{N}=0,15 \mathrm{eV}$ vào mô hình tính toán, kết quả đã chỉ ra rằng hai mức năng lượng trong khoảng $-4 \mathrm{eV}$ đã tách nhau ra một khoảng nhỏ. Như vậy, với ảnh hưởng của $\lambda_{N}$, các mức năng lượng thấp - đại diện cho các mức năng lượng trên

(a)

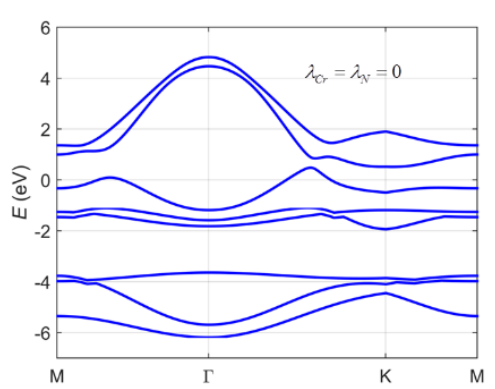

(c)

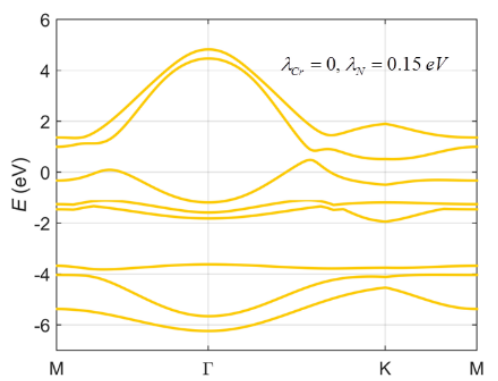

orbital phân lớp $p$ của $\mathrm{N}$ cũng được đẩy ra xa nhau. Tuy nhiên, với cùng một độ lớn của giá trị $\lambda$, dường như ảnh hưởng của SOC mạnh mẽ hơn trong các orbital ở phân lớp ngoài. Hay nói cách khác, ảnh hưởng của $\mathrm{SOC}$ trong các mức năng lượng của nguyên tử $\mathrm{Cr}$ mạnh hơn so với các mức năng lượng của N. Ngoài ra, khi đặt giá trị $\lambda_{C r}=\lambda_{N}=0,07 \mathrm{eV}$, kết quả thu được là cấu trúc vùng năng lượng của vật liệu (đường màu hồng) tương đối phù hợp với kết quả thu được bằng phương pháp khác (đường đứt nét màu đen) như thể hiện trên Hình $4 \mathrm{~d}$. Như vậy, có thể thấy rằng sự hiện diện của các giá trị $\lambda$ dường như không làm thay đổi hình dạng của các mức năng lượng trong cấu trúc vùng mà chỉ ảnh hưởng chủ yếu đến sự phân tách của các mức năng lượng trong cấu trúc điện tử đó.

(b)

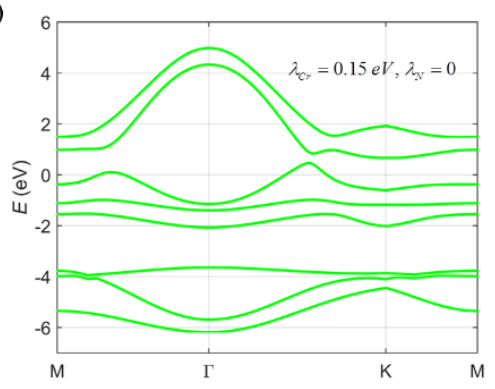

(d)

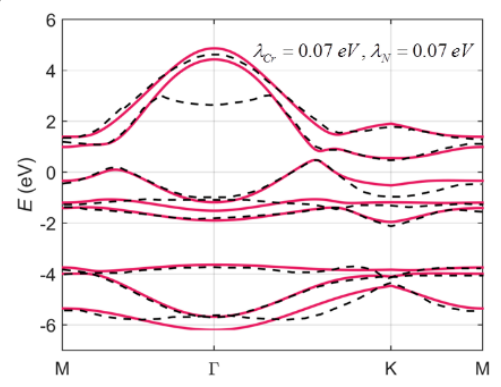

Hình 4. Cấu trúc vùng năng lượng của h-CrN với các giá trị khác nhau của SOC: (a) $\lambda_{C r}=\lambda_{N}=0$, (b) $\lambda_{C r}=0,15 \mathrm{eV}, \lambda_{N}=0$, (c) $\lambda_{C r}=0, \lambda_{N}=0,15 \mathrm{eV}$, (d) $\lambda_{C r}=\lambda_{N}=0,07 \mathrm{eV}$ và so sánh với kết quả từ nghiên cứu trước (đường đứt nét màu đen) (Luo et al., 2017)

\section{KẾT LUẬN VÀ ĐỀ XUẤT}

Trong bài báo này, mô hình tính toán được xây dựng và chỉ ra đóng góp của các orbital trong việc hình thành các tính chất điện tử đặc biệt của h-CrN, dựa trên phương pháp gần đúng liên kết mạnh và phương pháp luận hàm Green. Đồng thời, bộ tham số cấu trúc về năng lượng tương tác giữa các orbital của nguyên tử $\mathrm{Cr}$ và $\mathrm{N}$ cho mô hình phẳng và nhấp nhô cũng được trình bày. Kết quả nghiên cứu cho thấy rằng ở trạng thái nhấp nhô, cùng với sự đóng góp của các orbital nguyên tử $\mathrm{N}$ gần nhau nhất, trạng thái dẫn của vật liệu được kích thích thay đổi nhiều hơn so với trạng thái phẳng ban đầu. Đồng thời, tham số ảnh hưởng liên quan đến tính chất spin của vật liệu cũng được khảo sát. Đây là những kết quả bước đầu trong việc khảo sát tính chất điện tử của vật liệu $\mathrm{CrN}$ bằng phương pháp gần đúng liên kết mạnh, làm tiền đề để nghiên cứu những tính chất khác nhau của vật liệu dưới tác dụng của kích thích bên ngoài như điện trường, hướng tới ứng dụng trong tương lai. 


\section{LỜI CẢM TẠ}

Xin chân thành cảm ơn sự hỗ trợ kinh phí của Trường Đại học Kiên Giang cho đề tài cấp cơ sở, mã số A2020-KTCN-38.

\section{TÀI LIỆU THAM KHẢO}

Ali, M., Pi, X., Liu, Y., \& Yang, D. (2017). Electronic and magnetic properties of graphene, silicone and germanene with varying vacancy concentration. AIP Advances, 7(4), Article 045308, 1-11. http://doi.org/10.1063/1.4980836

Guzmán-Verri, G. G. \& Voon, L. C. L. Y. (2007). Electronic structure of silicon-based nanostructures. Physical Review B, 76(7), Article 075131, 1-10. https://doi.org/10.1103/PhysRevB.76.075131

Konschuh, S., Gmitra, M., \& Fabian, J. (2010). Tight-binding theory of the spin-orbit coupling in graphene. Physical Review B, 82(24), Article 245412, 1-11. https://doi.org/10.1103/PhysRevB.82.245412

Kuklin, A. V., Kuzubov, A. A., Kovaleva, E. A., Mikhaleva N. S., Tomilin F. N., Lee, H., \& Avramov, P.V. (2016). Two-Dimensional Hexagonal CrN with Promising Magnetic and Optical Properties: A Theoretical Prediction. Nanoscale, 9(2), 621-630. https://doi.org/10.1039/C6NR07790K

Luo, W., Xu, K., \& Xiang, H. (2017). Twodimensional hyperferroelectric metals: A different route to ferromagnetic-ferroelectric multiferroics. Physical Review B, 96(23), Article
$235415,1-7$.

https://doi.org/10.1103/PhysRevB.96.235415

Neto, A. H. C., Guinea, F., Peres, N. M. R., Novoselov, K. S., \& Geim, A. K. (2009). The electronic properties of graphene. Reviews of Modern Physics, 81(1), 109-162. https://doi.org/10.1103/RevModPhys.81.109

Novoselov, K. S., Fal'ko, V. I., Colombo, L., Gellert, P. R., Schwab, M. G., \& Kim, K. (2012). A roadmap for graphene. Nature, 490(7419), 192200. http://doi.org/10.1038/nature11458

Novoselov, K. S., Geim, A. K., Morozov, S. V., Jiang, D., Zhang, Y., Dubonos, S. V., Grigorieva, I. V., \& Firsov, A. A. (2004). Electric Field Effect in Atomically Thin Carbon Films. Science, 306(5696), 666-669. http://doi.org/10.1126/science.1102896

Takeda, K. \& Shiraishi, K. (1994). Theoretical possibility of stage corrugation in $\mathrm{Si}$ and $\mathrm{Ge}$ analogs of graphite. Physical Review B, 50(20), 14916-14922. https://doi.org/10.1103/PhysRevB.50.14916

Zhang. J., Zhao, B., Xue, Y., Zhou T., \& Yang, J. (2018). Coupling effect of topological states and Chern insulators in two-dimensional triangular lattices. Physical Review B, 97(12), Article 125403, 1-11. http://doi.org/10.1103/PhysRevB.97.125430

Zheng, F., Zhang, C. (2012). The electronic and magnetic properties of functionalized silicene: a first-principles study. Nanoscale Research Letters, 7(1), Article 422, 1-5 http://doi.org/10.1186/1556-276X-7-422 